\title{
A GENERAL COMPUTER PROGRAM FOR THE APPLICATION OF THE RIGOROUS BLOCK-ADJUSTMENT SOLUTION IN PHOTOGRAPHIC ASTROMETRY*
}

\author{
CHR. DE VEGT \\ Hamburger Sternwarte, F.R. Germany \\ and \\ H. EBNER \\ Universität Stuttgart, Institut für Photogrammetrie, F.R. Germany
}

\begin{abstract}
The evaluation of stellar positions from overlapping plates by rigorous block-adjustment methods leads to a very large system of normal equations containing both stellar positions and plate constants as unknowns. These general normal equations can be reduced to a subsystem with banded-bordered coefficient matrix, containing only the plate constants as unknowns. In the case of a large photographic net covering a hemisphere or sphere with multiple overlap of the plates, the actual number of unknown plate constants may exceed 20000, depending on the adopted reduction model.

The general structure of a computer program, now under development at the Hamburg observatory, is described in detail. The program, entirely written in Fortran IV language, is based on a very fast subroutine, developed at the university of Stuttgart, for the solution and inversion of large symmetric and positive-definite linear systems of equations with banded-bordered coefficient matrix. The adopted solution procedure in a generalized Cholesky method and the programmed algorithm is based on a direct transfer of submatrices instead of single coefficients of the system of equations during the solution process. The program provides a direct non-iterative solution of the block-adjustment problem with at least partial inversion of the normal equation matrix for evaluation of the standard deviations of the computed star positions at selected block-points.

Block-adjustment methods will provide maximum advantage with respect to error propagation and required minimum number of reference stars, if the number of unknown parameters is small.

The choice of suitable functional and stochastic reduction models and the application of the program for theoretical accuracy studies using inversion and simulation methods are discussed. The main aim of such theoretical error studies is to investigate the dependence of the positional accuracy obtained from the block-adjustment on the number, accuracy and distribution of reference stars and the adopted reduction model. Estimates of computing effort and necessary external storage capacity for the
\end{abstract}

* Paper in press, Monthly Notices Roy. Astron. Soc. 167 (1974). 
solution of the normal equations as a function of the number of unknowns and the actual matrix-structure are obtained.

For a variety of overlap patterns and block structures, occurring more frequently in photographic zone-work, simple analytic expressions for estimates of computing effort are derived. In addition, ordering schemes for an optimal arrangement of the single plates according to minimum bandwidth of the reduced normal equation matrix are provided.

Based on these results and adopting a CDC 7600 computer system, computing times and necessary external storage capacities are obtained for several large photographic nets, including a hemisphere and the full sphere with 2-fold and 4-fold overlap. In all cases a center-edge overlap pattern and a plate size of $5^{\circ} \times 5^{\circ}$ has been assumed. It is shown that a block-adjustment of the largest net, a full sphere with 4-fold overlap, could be provided within 3 hours computing time for the solution of the reduced normal equations containing 48000 unknown plate constants. 